\title{
Bile Acid Metabolism during Development: Metabolism of Lithocholic Acid in Human Fetal Liver
}

\author{
JAN GUSTAFSSON, STIG ANDERSON, AND JAN SJÖVALL \\ Departments of Pediatrics and Pharmaceutical Biochemistry, Uppsala University, Uppsala and Department of \\ Physiological Chemistry, Karolinska Institute, Stockholm, Sweden
}

\begin{abstract}
The metabolism of $\left[24-{ }^{14} \mathrm{C}\right]$ lithocholic acid was studied in the microsomal fraction of fetal human liver homogenates. Fetal livers were obtained at legal abortions performed between wk 14 and 24. Product formation was analyzed by thin-layer chromatography, liquid chromatography, and gas chromatography-mass spectrometry. From wk 14 lithocholic acid was hydroxylated in several positions. Hyodeoxycholic acid $(3 \alpha, 6 \alpha$-dihydroxy-5 $\beta$-cholanoic) and $1 \beta, 3 \alpha$-dihydroxy-5 $\beta$-cholanoic acid were identified among the products. The $3 \beta$-isomer of the former acid, $6 \alpha-$ hydroxy-3-oxo-5 $\beta$-cholanoic acid and a 2-hydroxylithocholic acid were tentatively identified. Two other metabolites carried a hydroxyl group in an unknown position, one of them probably at an angular methyl group. The highest total conversions per $\mathrm{mg}$ of microsomal protein were obtained with preparations from 18 wk fetuses. The results are discussed with particular reference to the role of lithocholic acid in fetal hepatotoxicity. (Pediatr Res 21: 99103, 1987)
\end{abstract}

\section{Abbreviations}

GLC, gas-liquid chromatography

GC/MS, gas chromatography-mass spectrometry

In humans the primary bile acids, cholic acid $(3 \alpha, 7 \alpha, 12 \alpha-$ trihydroxy- $5 \beta$-cholanoic acid) and chenodeoxycholic acid $(3 \alpha$, $7 \alpha$-dihydroxy-5 $\beta$-cholanoic acid) are excreted in bile as glycine and taurine conjugates (1). Deconjugation and dehydroxylation by intestinal microorgansims yield the secondary bile acids deoxycholic acid $(3 \alpha, 12 \alpha$-di-hydroxy- $5 \beta$-cholanoic acid) and lithocholic acid ( $3 \alpha$-hydroxy-5 $\beta$-cholanoic acid) (1). Deoxycholic acid is efficiently reabsorbed and returned to the liver where it is conjugated and to some extent hydroxylated (1). Lithocholic acid also is reabsorbed but less efficiently since it is poorly soluble in water and tightly bound to intestinal microorganisms (1).

Little information is available on the metabolism of primary and secondary bile acids during fetal life (2). There is evidence that human fetal liver can conjugate primary bile acids (2). Due to lack of microflora there is no formation of secondary bile acids (2). The occurence of deoxycholic and lithocholic acids in meconium has earlier been thought to reflect placental transfer of these compounds (2), but at least in case of lithocholic acid a primary fetal synthesis from cholesterol cannot be excluded (3).

Received February 2, 1986; accepted September 10, 1986.

Correspondence and reprint requests, Jan Gustafsson M.D., Ph.D., Department of Pediatrics, University Hospital, S-751 85 Uppsala, Sweden.

Supported by the Swedish Medical Research Council (Projects 19P-07514, 03X218, and 03X-219) the "Expressin" Prenatal Research Foundation and the Swedish Society of Medical Sciences.
We have shown recently that fetal liver efficiently metabolizes taurodeoxycholic acid by hydroxylations at the $1 \beta$ - and $7 \alpha-$ positions (4).

Lithocholic acid is a potent toxic compound causing cholestasis in many species (5). A role for lithocholic acid in the pathogenesis of neonatal cholestasis/extra-hepatic biliary atresia has been suggested (6). Presence of sulfated conjugates of lithocholic acid in meconium indicates that sulfation may represent one mechanism for elimination of this bile acid in the fetus $(6$, 7).

The present work reports on the metabolism of lithocholic acid by hydroxylation in the microsomal fraction of human fetal liver.

\section{MATERIALS AND METHODS}

Materials. $\left[24-{ }^{14} \mathrm{C}\right]$ lithocholic acid (specific radioactivity, 55 $\mathrm{Ci} / \mathrm{mol}$ ) was obtained from Amersham, Buckinghamshire, England. Before use the material was purified by thin-layer chromatography with solvent system S 11 (8). Lithocholic, chenodeoxycholic, deoxycholic, and hyodeoxycholic $(3 \alpha, 6 \alpha$-dihydroxy- $5 \beta$-cholanoic) acids were obtained from Sigma Chemical Co., St. Louis, MO and NADPH was obtained from Sigma Chemical Co. Solvents were of reagent grade and redistilled. Diazomethane was prepared according to Schlenk and Gellerman (9). Pyridine, hexamethyldisilazane, and trimethylchlorosilane were redistilled. Unisil was from Clarkson Chem. Co (Williamsport, PA), SP-Sephadex from Pharmacia Fine Chemicals (Uppsala, Sweden), and Lipidex-DEAP from Packard Instr. Co. (Downers Grove, IL). The two ion exchangers were washed with aqueous ethanol and ethanol prior to use. GLC was carried out on $25 \mathrm{~m} \times 0.32 \mathrm{~mm}$ (i.d.) fused silica capillary columns coated with OV-1 $(0.20 \mu \mathrm{m}$ film thickness, Orion Analytica Espoo, Finland) or cross-linked methyl silicone $(0.25 \mu \mathrm{m}$ film thickness, Quadrex Corp., New Haven, CT) in a Carlo Erba HRGC 4160 gas chromatograph. Helium was used as carrier gas at 50-100 $\mathrm{kPa}$ and column temperatures were $270-290^{\circ}$.

Experimental. Nine human fetuses were obtained at legal abortions performed between wk 14 and 24 (c.f. Table 2). Consent was given by the ethical committee of the University of Uppsala. The abortions were performed with prostaglandins or via hysterotomy. Fetal age was determined from measurements of fetal height and from data concerning the pregnancies. After abortion, the fetuses were taken to the laboratory and liver tissue was taken out and chilled in ice-cold buffer solution. Liver homogenates $(10 \%, \mathrm{w} / \mathrm{v})$ were prepared within $45-60 \mathrm{~min}$ in $0.25 \mathrm{M}$ sucrose using a Potter-Elvehjem homogenizer equipped with a loosely fitting pestle (10). The microsomal fraction was prepared by centrifugation of the homogenate at $800 \times g, 20,000$ and $100,000 \times g(10)$. The final pellet was suspended in onefourth to one-half of the initial volume of $0.05 \mathrm{M}$ Tris-acetate 
buffer, pH 7.4, containing $0.1 \mathrm{mM}$ EDTA, and $20 \%$ (v/v) glycerol. Microsomal protein was $0.9-3 \mathrm{mg} / \mathrm{ml}$ when determined according to Bradford (11). The lack of detectable 26-hydroxylase activity toward $5 \beta$-cholestane- $3 \alpha, 7 \alpha, 12 \alpha$-triol in the microsomal fraction indicates absence of significant contamination with mitochondrial protein in the microsomal fraction $(c . f$. Reference 12). With regard to peroxisomal protein some degree of contamination was found. Thus, the activity of urate oxidase, a peroxisomal marker enzyme (13), was about $15 \%$ of that in whole liver homogenate.

Incubation procedure and analysis of incubation mixtures. [24$\left.{ }^{14} \mathrm{C}\right]$ lithocholic acid was diluted with nine parts unlabeled lithocholic acid and $0.25 \mu \mathrm{mol}$ (specific radioactivity $5.5 \mathrm{Ci} / \mathrm{mol}$, corresponding to about 8.5 atom percent excess of ${ }^{14} \mathrm{C}$ at $\mathrm{C}-24$ ) was added in $40 \mu \mathrm{l}$ of acetone. Of microsomal fraction $1.0 \mathrm{ml}$ was used and total incubation volume was $2 \mathrm{ml}$. NADPH, 2 $\mu \mathrm{mol}$, was added and incubations were performed for 20-40 min at $37^{\circ} \mathrm{C}$ and were terminated by addition of $95 \%(\mathrm{v} / \mathrm{v})$ aqueous ethanol. After acidification, the mixtures were extracted twice with diethyl ether. The combined ether extracts were washed with water until neutral and taken to dryness. Less than $1 \%$ of the incubated radioactivity remained in the water phases. The residue was subjected to thin-layer chromatography using solvent system S 11 (8). Chenodeoxycholic, deoxycholic, and hyodeoxycholic acids were used as external standards. Product formation was determined by radioactivity scanning of the thin-layer chromatograms. For identification, zones containing radioactive products were scraped into a $0.8 \mathrm{~cm}$ id column on top of $2 \mathrm{~cm}$ of Unisil and eluted with $25 \mathrm{ml}$ of water-saturated ethyl acetate. The extract was taken to dryness, dissolved in $5 \mathrm{ml} 70 \%(\mathrm{v} / \mathrm{v})$ aqueous ethanol and passed through a column of SP-Sephadex $\left(40 \times 4 \mathrm{~mm}\right.$ in $\mathrm{H}^{+}$-form $)$and a column of Lipidex-DEAP $(20 \times$ $4 \mathrm{~mm}$ in acetate form) (14). After washing with $10 \mathrm{ml} 70 \%$ ethanol, the bile acids were eluted from the latter column with 8 $\mathrm{ml} 0.1 \mathrm{M}$ acetic acid in $70 \%$ ethanol. After methylation with diazomethane, they were trimethylsilylated in pyridine/hexamethyldisilazane/trimethylchlorosilane, $3: 2: 1(\mathrm{v} / \mathrm{v} / \mathrm{v})$, at $60^{\circ} \mathrm{C}$, $30 \mathrm{~min}$. The derivatives were analyzed by GLC. GC/MS was carried out on a VG 7070E instrument (VG Analytical Ltd, Manchester, England) with the capillary columns directly introduced to the ion source, The electron energy was $22.5 \mathrm{eV}$.

In other experiments, the entire incubations were added to 50 $\mathrm{ml}$ of ethanol and heated at $60^{\circ} \mathrm{C}$ for $1 \mathrm{~h}$. After filtration, the residue was extracted once again with $50 \mathrm{ml}$ of ethanol. The combined extract was taken to dryness and the residue was partitioned between $0.1 \mathrm{M}$ hydrochloric acid $(20 \mathrm{ml})$ and ethyl acetate $(50 \mathrm{ml})$. The water phase was extracted once more with ethyl acetate $(50 \mathrm{ml})$, the combined extracts were washed with small portions of water and taken to dryness. The residue was dissolved in $20 \mathrm{ml} 90 \%$ ethanol and a bile acid fraction was isolated, derivatized, and analyzed by GLC and GC/MS as described above. Samples in which lithocholic acid was added after termination of the incubations were analyzed as controls in the same way.

\section{RESULTS}

Incubation of $\left[24-{ }^{14} \mathrm{C}\right]$ lithocholic acid with the microsomal fraction of fetal liver and NADPH gave three radioactive zones on thin-layer chromatography. In the active preparations the relative conversions were: $0.1-4.2 \%$ (zone I), $0.2-3.6 \%$ (zone II), and $0.1-3.6 \%$ (zone III). The products migrated as dihydroxycholanoic acids with mean $R_{r}$-values (migration of the product divided by migration of the solvent front) for the zones of 0.27 (zone I), 0.38 (zone II), and 0.53 (zone III), respectively. With the solvent system used the mobility of zone I corresponded to that of hyodeoxycholic acid (reference) and the mobility of zone II to that of chenodeoxycholic acid (reference). Except for the radioactivity found in zones I-III, all the remaining radioactivity migrated as lithocholic acid (reference). The gas chromatographic analyses of the incubation mixtures and the eluates of the thinlayer chromatographic zones indicated formation of several metabolites.

The GLC and GC/MS analyses of the crude incubation mixtures were difficult to interpret due to many overlapping peaks. However, besides lithocholic acid only cholic acid could be detected in the control experiments where lithocholic acid was added after termination of the incubation. The amount of cholic acid was $1-2 \%$ of that of the lithocholic acid. In contrast, the analyses of microsomal fractions incubated with lithocholic acid indicated formation of several dihydroxy bile acids which were studied after separation by thin-layer chromatography. The analyses of material from the thin-layer chromatograms also showed complex mixtures except in the case of zone I where hyodeoxycholic acid predominated. In all cases the origin of a compound from added lithocholic acid was definitively established by calculation of the percentage of ${ }^{14} \mathrm{C}$-labeled molecules from the isotope pattern of suitable fragment ions in the mass spectra. About $8.5 \%$ of the incubated lithocholic acid contained ${ }^{14} \mathrm{C}$. This abundance of heavy atoms was also seen in the spectra of the metabolites and could be determined with an accuracy of about $\pm 20 \%$. Cholic acid, also detected in the analyses, did not contain ${ }^{14} \mathrm{C}$. Retention indices and mass spectral characteristics of the seven metabolites detected are given in Table 1.

The retention index and mass spectrum of the derivative of the major product in zone I were identical with those of the derivative of hyodeoxycholic acid (Fig. 1A). Prominent ions at $\mathrm{m} / \mathrm{z} 405$ (M-145, i.e. loss of C-1-C-4) and at $\mathrm{m} / \mathrm{z} 323$ (consisting of side chain, D-ring, C-8, C-7, and C-6 with its trimethylsiloxy group) are particularly characteristic for the 3,6-bis-trimethylsiloxy structure (c.f. Reference 15). A minor compound with a slightly longer retention time gave a very similar mass spectrum, showing that it was the derivative of an isomer of hyodeoxycholic acid. The $6 \beta$-isomer could be excluded since its derivative had a shorter retention time than that of hyodeoxycholic acid. This indicates a $3 \beta$-configuration of the metabolite. In the sample analyzed by GC/MS this compound constituted about $15 \%$ of the bile acids in zone I.

Zone II contained at least three metabolites occurring in similar amounts in the sample analyzed by GC/MS. The derivative of one of these (RI 3285) gave a mass spectrum with an intense base peak at $\mathrm{m} / \mathrm{z} 217$ typical of a 1,3-bis-trimethylsiloxy structure (Fig. $1 B)(4,14)$. Peaks at $m / z 142$ and 143 were also present (16). A peak of low intensity at $\mathrm{m} / \mathrm{z} 535(\mathrm{M}-15)$ and peaks at $m / z 460(\mathrm{M}-90)$ and $370(\mathrm{M}-2 \times 90)$ showed the presence of two hydroxyl groups. The retention index (3285) compared with that of the derivative of $1 \beta, 3 \alpha, 12 \alpha$-trihydroxy-5 $\beta$-cholanoic acid (RI 3293) indicated that the compound was the same as that previously obtained by hydroxylation of lithocholic acid with a $1 \beta$-hydroxylating Penicillium species (16). Thus, this metabolite is identified as $1 \beta, 3 \alpha$-dihydroxy- $5 \beta$-cholanoic acid.

The derivative of another bile acid from zone II (RI 3254) gave a mass spectrum with a base peak at $m / z 143$ and an intense ion at $\mathrm{m} / z 142$ (Fig. 1C). This is typical for steriods with a 2,3bis-trimethylsiloxy structure which is supported by the presence of an ion at $m / z 318$ corresponding to loss of C-1-C-4 with two trimethylsiloxy groups (M-232). A molecular ion was not observed but the intensity of $m / z 460$ (corresponding to M-90) and the thin-layer chromatographic behavior indicate that the metabolite is a dihydroxy acid. It is tentatively identified as $2 \xi, 3 \alpha-$ dihydroxy- $5 \beta$-cholanoic acid.

The derivative of a third metabolite in zone II gave an intense base peak at $\mathrm{m} / \mathrm{z} 357$, corresponding to M-90-103 (Fig. 1D). Loss of a fragment of mass 103 is seen in spectra of trimethylsilyl ethers of steroids with a derivatized primary hydroxyl group. The combined presence of ions at $\mathrm{m} / z 460,370$, and 255 indicates a side chain without a hydroxyl group. Thus, hydroxylation at C18 or C-19 seems likely. The peak at $m / z 249$, if significant (c.f. Reference 15), favors C-19. There was no evidence of any formation of chenodeoxycholic acid from lithocholic acid. 
Table 1. Retention indices and mass spectral characteristics of methyl ester trimethylsilyl ethers of bile acids formed after incubation of lithocholic acid with preparations from human fetal liver

\begin{tabular}{|c|c|c|c|}
\hline RI* & TLC $\dagger$ & Masses, $m / z$, and relative intensities (\%) of major ions & Bile acid identified \\
\hline 3225 & I & $\begin{array}{l}550,460,405,370,331,323,315,303,263,255,249,228 \\
(7)(24)(21)(100)(12)(32)(18)(10)(14)(31)(11)(20) \\
213,161,143,129 \\
(31)(26)(10)(27)\end{array}$ & Hyodeoxycholic acid \\
\hline 3238 & I & $\begin{array}{l}550,460,405,370,331,323,315,303,263,255,249,228, \\
(7)(48)(49)(100)(15)(24)(9)(7)(7)(20)(14)(9) \\
213,161,143,129 \\
(16)(20)(34)(20)\end{array}$ & $3 \beta, 6 \alpha$-dihydroxy-5 $\beta$-cholanoic acid $\ddagger$ \\
\hline 3254 & II & $\begin{array}{l}460,431,370,339,334,331,318,262,255,249,228,213 \\
(88)(10)(50)(12)(34)(34)(15)(7)(31)(18)(10)(24) \\
196,171,143,142,129 \\
(16)(21)(100)(69)(39)\end{array}$ & $2 \xi, 3 \alpha$-dihydroxy- $5 \beta$-cholanoic acid $\$$ \\
\hline 3285 & II & 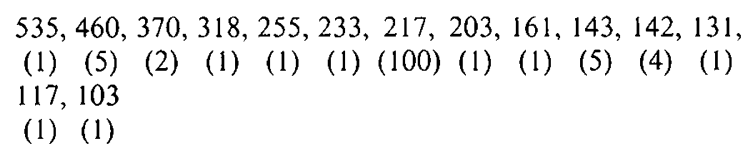 & $1 \beta, 3 \alpha$-dihydroxy-5 $\beta$-cholanoic acid \\
\hline 3293 & III & $\begin{array}{l}476,461,386,371,368,323,316,313,271,253,249,244 \\
(4)(33)(42)(15)(8)(54)(12)(12)(100)(13)(14)(15) \\
229,129 \\
(22)(22)\end{array}$ & $6 \xi$-hydroxy-3-oxo- $5 \beta$-cholanoic acid \\
\hline 3326 & II-III & 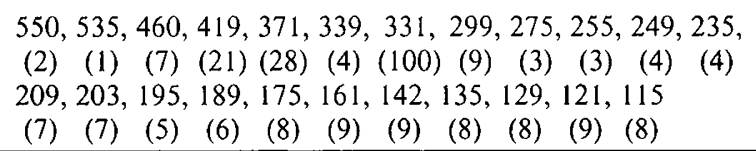 & Unknown dihydroxy bile acid \\
\hline
\end{tabular}

* Retention index (RI) on a fused silica capillary column coated with a cross-linked methyl silicone (Quadrex, column temperature $280^{\circ}$ ). The derivative of cholic acid had an RI of 3216 under the same conditions. The retention index expresses retention time in relation to the retention times of a series of $\mathrm{n}$-paraffins, e.g. 3200 corresponds to the retention time of dotriacontane.

$\dagger$ Thin-layer chromatography (TLC) zone containing the compound.

$\ddagger$ Tentative structure, see text.

Two metabolites were found in zone III of the thin-layer chromatogram. The derivative of one of these (RI 3293) gave a mass spectrum with a molecular ion at $\mathrm{m} / z 476$ corresponding to a methyl cholanoate with one trimethylsiloxy and one oxo group. The intense peak at $\mathrm{m} / \mathrm{z} 323$ (see above) and a peak at $m / z 316$ (386-70, i.e. loss of A-ring with oxo group) indicate that this metabolite was 6 -hydroxy-3-oxo- $5 \beta$-cholanoic acid.

The second metabolite in zone III had a lower mobility as indicated by its presence also in zone II. The derivative gave a mass spectrum with prominent ions corresponding to losses of fragments of mass $131(\mathrm{~m} / \mathrm{z} 419, \mathrm{M}-131), 129(\mathrm{~m} / \mathrm{z} 331$, M-90$129)$, and $89(\mathrm{~m} / z$ 371, M-90-89). A molecular ion showed that two trimethylsiloxy groups were present and the peak at $m / z 115$ indicated an unsubstituted side chain. The structure remains unknown; it is likely that hydroxylation has occurred at a position close to C-3. In the sample analyzed by GC/MS this compound constituted about $75 \%$ of the metabolites identified in zone III.

Due to the complexity of the metabolite mixture and the low concentrations, the individual compounds could not be accurately quantified. However, most of the radioactivity in zone I was due to hyodeoxycholic acid and its isomer.

There was no continuous increase in conversion of lithocholic acid in relation to increasing gestational length (Table 2). Instead, the highest total conversions were obtained with three of four preparations from fetuses in wk 18 .

\section{DISCUSSION}

Lithocholic acid, irrespective of its origin from the mother or the fetus, is potentially hepatotoxic to the fetus (7). Several pieces of evidence indicate that the fetus may detoxify lithocholic acid by sulfation or by excretion across the placenta (7). The present study shows that human fetal liver microsomes have the capacity for hydroxylation of lithocholic acid in $1 \beta$ - and $6 \alpha$-positions and most likely at $\mathrm{C}-2$ and at an angular methyl group. Under the conditions used there was also oxidoreduction at C-3.

As mentioned above there was no correlation between gestational age and conversion. The marked variation between preparations from different fetal livers may in part be due to enzyme inactivation during the abortion and work-up procedure (c.f. Reference 12).

The results should be compared to those of studies on bile acid metabolism in adult human liver. In such studies Björkhem et al. (17) found no microsomal hydroxylation of free or conjugated lithocholic acid, whereas Trülsch et al. (18) reported the existence of microsomal $6 \alpha$-hydroxylase activity toward taurolithocholic acid in liver biopsies. It should be pointed out that failure to detect certain $5 \beta$-cholanoic acid hydroxylase activities in the adult human liver in vitro does not necessarily mean a complete absence of such enzyme activities. In fact, the presence of small amounts of 1- and 6-hydroxylated bile acids in urine of normal (14) and cholestatic subjects $(14,19,20)$ speaks in favor of the 

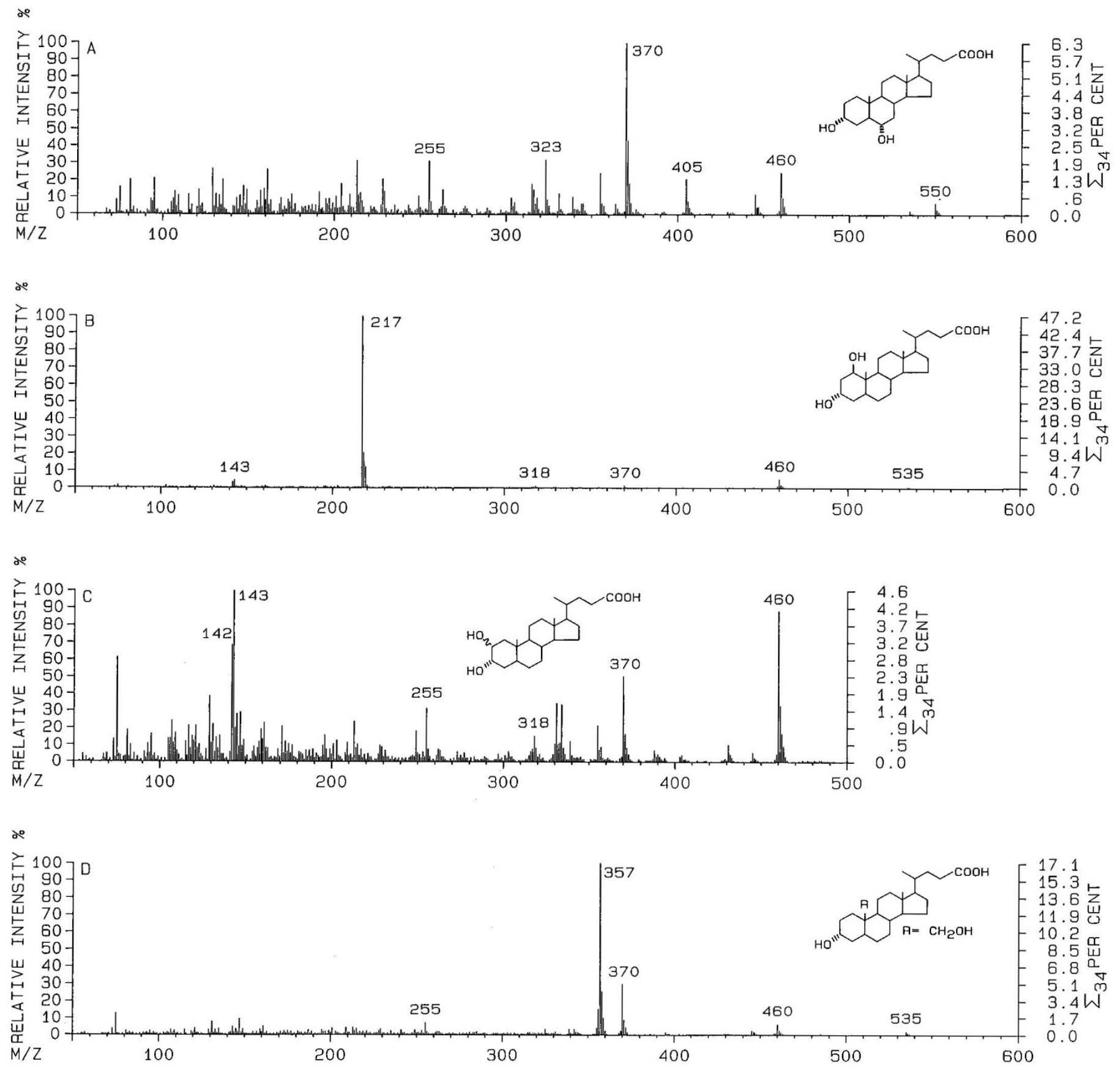

Fig. 1. Mass spectra of the methyl ester trimethylsilyl ethers of four hydroxylated metabolites of lithocholic acid. $A$, derivative of $3 \alpha, 6 \alpha-$

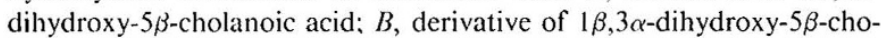

lanoic acid; $C$, derivative of tentatively identified $2 \xi, 3 \alpha$-dihydroxy- $5 \beta$ cholanoic acid; $D$, derivative of dihydroxy-cholanoic acid possibly hydroxylated at C-19 (see text).

Table 2. Hydroxylation of lithocholic acid by microsomal preparations from livers of fetuses of different gestational ages*

\begin{tabular}{|c|c|c|c|c|c|c|c|c|c|}
\hline & \multicolumn{9}{|c|}{ Gestational wk } \\
\hline & 14 & 15 & 17 & 18 & 18 & 18 & 18 & 24 & 24 \\
\hline & \multicolumn{9}{|c|}{ (Product formation $\mathrm{pmol} / \mathrm{mg}$ protein $\mathrm{x} \min$ ) } \\
\hline Products in zone II & 21 & 15 & $<10$ & 138 & 48 & $<10$ & 21 & 11 & 10 \\
\hline Products in zone III & 15 & 10 & $<10$ & $<10$ & 69 & $<10$ & 47 & 36 & 23 \\
\hline
\end{tabular}

* Incubations were performed as described in "Materials and methods" using $0.25 \mu \mathrm{mol}$ of lithocholic acid in each incubation. Every incubation corresponds to one fetal liver microsomal preparation. 
existence of corresponding hydroxylase activities in liver. However, the quantitative importance of such hydroxylations cannot be estimated from the rate of excretion of the corresponding bile acids in urine, since renal clearance for such bile acids apparently is high (20). The formation of 1-, 2-, and 6-hydroxylated bile acids in fetal liver is also in agreement with work by Strandvik and Wikström (21) on tetrahydroxylated bile acids in urine of newborn infants.

Back and Walter (22) have described a pattern of "immature" bile acids in meconium. The excretion of immature bile acids in urine of cholestatic subjects has led to the suggestion that cholestasis induces a return to a fetal stage of metabolism (22). To establish whether a fetal mode of bile acid metabolism exists under such conditions, it would be necessary to study hydroxylase activities in liver preparations from cholestatic subjects.

Lithocholic acid appears to be most toxic in species unable to convert it into more polar compounds (17). Conjugation with glycine or taurine does little to decrease the toxic potential of lithocholic acid (23), but sulfation $(6,7)$ and hydroxylation may represent ways for detoxification and elimination of this bile acid. Such metabolic transformations make this nonpolar compound more water soluble and promote its excretion (7).

The formation of 6-hydroxy-3-oxo-5 $\beta$-cholanoic and $3 \beta$-hydroxy- $5 \beta$-cholanoic acids from lithocholic acid in fetal liver is interesting in view of earlier work on lithocholic acid metabolism (17). Thus, Björkhem et al. (17) reported that an adult liver homogenate was capable of converting lithocholic acid into $3 \beta$ hydroxy- $5 \beta$-cholanoic acid (isolithocholic acid) with the intermediate formation of 3 -oxo- $5 \beta$-cholanoic acid. Since the aim was to identify hydroxylated products, a possible isomerization of lithocholic acid was not investigated. Isolithocholic acid may well have been formed in the incubations, but under the conditions used for thin-layer chromatography lithocholic acid will not separate from $3 \beta$-hydroxy- $5 \beta$-cholanoic acid. The lithocholic acid zone was not analyzed by GC/MS. Epimerization of the $3 \alpha$-hydroxyl group may have physiological implications since it has been reported that $3 \beta$-hydroxysteroids are less toxic regarding hemolytic activity than $3 \alpha$-hydroxysteriods (24). Further, isolithocholic acid is less active than lithocholic acid with respect to pyrogenic activity (25).

The present results suggest that the fetus is more capable than the adult of eliminating lithocholic acid by way of hydroxylations at several positions of the $\mathrm{C}_{24}$-molecule. Such metabolism could be particularly important in recurrent cholestasis of pregnancy, a condition known to be associated with elevated fetal bile acid levels (26).

The absence or low amounts of secondary bile acids during the first years of life have been explained by the fact that there is a slow colonization of intestinal microorganisms in the infant (1). However, metabolism of lithocholic acid by hydroxylation may also explain the small amounts of lithocholic acid in this period of life as has been previously suggested in the case of deoxycholic acid (4).

If lithocholic acid plays a role in the initiation of neonatal liver disease $(6,27)$, such conditions could be associated with impaired fetal metabolism of lithocholic acid. It would therefore be of interest to compare lithocholic acid metabolism in microsomal preparations from infants with extrahepatic biliary atresia to that in preparations from infants without liver disease.
Acknowledgments. The skillful technical assistance of Miss Åsa Byström and Mrs. Marianne Bengtsberg is gratefully acknowledged.

\section{REFERENCES}

1. Danielsson H, Gustafsson J 1981 Biochemistry of bile acids in health and disease. In: Joachim HL (ed) Pathobiology Annual, Vol XI. Raven Press, New York, pp 259-298

2. Subbiah MTR, Hassan AS 1982 Development of bile acid biogenesis and its significance in cholesterol homeostasis. Adv Lipid Res 19:137-161

3. Lester R, St. Pyrek J, Adcock EW 1983 Diversity of bile acids in the fetus and newborn infant. J Pediatr Gastroenterol Nutr 2:355-364

4. Gustafsson J Andersson S, Sjövall J 1985 Bile acid metabolism during development: metabolism of taurodeoxycholic acid in human fetal liver. Biol Neonate 47: 26-41

5. Tuchweber B, Perea A, Lee D, Yousef IM 1983 Lithocholic acid induced cholestasis in newborn rats. Toxicol Lett 19:107-112

6. Chen LJ, Thaler MM, Bolt RJ, Golbus MS 1978 Enzymatic sulfation of bile salts: III. Enzymatic sulfation of taurolithocholate in human and guinea pig fetuses and adults. Life Sci 22:1817-1820

7. Watkins JB, Brown ER 1977 Conjugation and sulfation of lithocholic acid in fetal liver. In: Paumgartner G, Stiehl A (eds) Bile acid metabolism in health and disease. MTP Press Limited, Lancaster, pp 65-70

8. Eneroth P 1963 Thin-layer chromatography of bile acids. J Lipid Res 4:11-16

9. Schlenk H, Gellerman JL 1960 Esterification of fatty acids with diazomethane on a small scale. Anal Chem 32:1412-1414

10. Bjökhem I, Gustafsson J $1973 \omega$-Hydroxylation of steroid side-chain in biosynthesis of bile acids. Eur J Biochem 36:201-212

11. Bradford $M 1976 \mathrm{~A}$ rapid and sensitive method for the quantitation of microgram quantities of protein utilizing the principle of protein-dye binding. Anal Biochem 72:248-254

12. Gustafsson J 1986 Bile acid biosynthesis during development. Hydroxylation of $\mathrm{C}_{27}$-sterols in human fetal liver. $\mathrm{J}$ Lipid Res In press

13. Schneider WC. Hogeboom GH 1952 Intracellular distribution of enzymes. IX Certain purine-metabolizing enzymes. J Biol Chem 195:16!-166

14. Almé B, Bremmelgaard A, Sjövall J, Thomassen P 1977 Analysis of metabolic profiles of bile acios in urine using a lipophilic anion exchanger and computerized gas-liquid chromatography-mass spectrometry. J Lipid Res 18:339-362

15. Sjövall J, Lawson AM, Setchell KDR 1985 Mass spectrometry of bile acids. Meth Enzymol 111:63-113

16. Carlström K, Kirk DN, Sjövall J 1981 Microbial synthesis of $1 \beta$ - and $15 \beta$ hydroxylated bile acids. J Lipid Res 22:1225-1234

17. Björkhem I, Einarsson K, Hellers G 1973 Metabolism of mono- and dihydroxylated bile acids in preparations of human liver. Eur J Clin Invest 3:459465

18. Trülsch D, Roboz J, Greim H, Czygan P, Rudick J, Hutterer F, Schaffner F, Popper H 1974 Hydroxylation of taurolithocholatc by isolated human liver microsomes. Biochem Med 9:158-166

19. Bremmelgaard A, Sjövall J 1979 Bile acid profiles in urine of patients with liver diseases. Eur J Clin Invest 9:341-348

20. Bremmelgaard A, Sjövall J 1980 Hydroxylation of cholic, chenodeoxycholic and deoxycholic acids in patients with intrahepatic cholestasis. J Lipid Res 21:1072-108!

21. Strandvik B, Wikström S-Ä 1982 Tetrahydroxylated bile acids in healthy human newborns. Eur J Clin Invest 12:301-305

22. Back P, Walter K 1980 Developmental pattern of bile acid metabolism as revealed by bile acid analysis of meconium. Gastroenterology 78:671-676

23. Balistreri WF, Suchy FJ, Farell MK, Heubi JE 1981 Pathologic versus physiologic cholestasis: Elevated serum concentration of a secondary bile acid in the presence of hepatobiliary disease. J Pediatr 98:339-402

24. Berliner F. Schoenheimer R 1938 Hemolytic and antihemolytic properties of bile acids and sterols in relation to their structure. $\mathrm{J}$ Biol Chem 124:525-541

25. Norman A, Palmer RH 1964 Metabolities of lithocholic acid-24- ${ }^{14} \mathrm{C}$ in human bile and feces. J Lab Clin Med 63:986-1001

26. Laatikainen TJ, Lehtonen PJ, Hesso AE 1978 Fetal sulfated and nonsulfated bile acids in intrahepatic cholestasis of pregnancy. J Lab Clin Med 92:185193

27. Jenner RE, Howard ER 1975 Unsaturated monohydroxy bile acids as a cause of idiopathic obstructive cholangiopathy. Lancet 2:1073 